\title{
Crop Water Condition Mapping by Optical Remote Sensing
}

\author{
Wojtaszek, M. V. ${ }^{1}$ and Abdurahmanov, $\mathbf{I}^{2}$ \\ ${ }^{1}$ Institute of Geoinformatics, Alba Regia Faculty of Engineering, Óbuda University, Székesfehérvár, Hungary \\ E-mail: wojtaszek.malgorzata@amk.uni-obuda.hu \\ ${ }^{2}$ Tashkent Institute of Irrigation and Agricultural Mechanization Engineers, Kari Niyazov str., 39, 100000 \\ Tashkent, Uzbekistan
}

\begin{abstract}
Crop water stress monitoring represents a fundamental step in agricultural production. In order to increase water savings and enhance agricultural sustainability, implementation of suitable irrigation scheduling methods is essential, and requires early detection of water stress in crops, before it causes irreversible damage and yield loss. There are different methods to measure water stress, some of them are based on soil moisture measurements while others are based on calculations of vegetation indices, evapotranspiration or soil water balance. Currently, the use of remote sensing technologies for the analysis of plant water status comprises a wide range of available methods such as infrared thermometry for canopy temperature measures, microwave radiation for soil water content assessment, and spectral vegetation indices for the study of the reflectance responses of canopies to different environmental conditions. The aim of the presented work is to investigate the applicability of the optical trapezoid model (OPtical TRApezoid Model) in mapping the moisture content within agricultural field. The model ability to provide vegetation characteristics, and crop water status at the canopy scale can improve the site-specific decision-making process in a precision agriculture.
\end{abstract}

\section{Introduction}

Growth and reproduction of crops strongly depends on their environment, and any change in environmental conditions that determines a shift from the crop's optimal state can be considered as stressful. Water is a very important factor in agriculture and plays an essential role in crop production throughout the growing season. Water is required for the germination of seeds and as soon as growth starts water serves as a carrier in the distribution of mineral nutrients. Biomass production is inseparably connected with water demand. Many of the biochemical reactions that are part of growth occur in water or water itself participates in the reactions, e.g: respiration, photosynthesis. Plant available water is absorbed from the root zone in support to the transpiration process, and it also facilitates the absorption of nutrients through their roots. Transpiration controls also the plant's canopy temperature as it does not heat or cool too quickly, and cools plants by evaporating from the leaf surface. Transpiration leads to water loss, and when the soil water reserve is not enough to satisfy the plant's transpiration needs, the photosynthesis and growth of plants will stop in response to the water stress. This results in a reduction in plant yield. The transpiration rate is influenced by the relative humidity of the surrounding air, air temperature and wind speed. During the vegetation-free period the soil is also exposed to water loss. The evaporation from soil is largely determined by the amount of solar radiation reaching the soil surface. A number of studies (Wang et al., 2015 and Ghulam et al., 2007) indicate that water stressed crops have reduced evapotranspiration, and manifest other symptoms such as leaf wilting, stunted growth, and leaf area reduction. Also, water stress adversely affects the physiological and nutritional development of crops, leading to reduced biomass, yield, and quality of crops.

Water is a vital component in the functioning of plants and soil moisture is the dominant factor controlling its supply (Ghulam et al., 2007). So that information on water stress conditions and their analysis is necessary to achieve optimal outputs. Crop water stress monitoring represents a fundamental step in agricultural production, measures of plant water status are required to better understand the mechanisms of plant response and adaptation to water stress, and for the optimisation of crop production (Osakabe et al., 2014) through precision irrigation. In order to increase water savings and enhance agricultural sustainability, implementation of suitable irrigation scheduling methods is essential (Osakabe et al., 2015), and requires early detection of water stress in crops, before it causes irreversible damage and yield loss. 
There are different methods to measure water stress, some of them are based on soil moisture measurements while others are based on calculations of vegetation indices, evapotranspiration or soil water balance. Conventional methods for monitoring crop water stress rely on in situ soil moisture measurements and meteorological variables to estimate the amount of water lost from the plant-soil system during a given period (González-Dugo et al., 2006). Other methods of detecting plant water status involve soil water balance calculations, direct and indirect measurement of plant water status, via stomatal conductance and leaf water potential. The disadvantage of measurements based on soil sampling is that these methods are time consuming and produce point information. These approaches are time consuming, labour intensive and unsuitable for automation, due to heterogeneity of soil and crop canopy. Those methods usually provide point information that give poor indications of the overall status of the field and are difficult to up-scale to the plant, field or regional level (Jones, 2012, Ihuoma and Madramootoo, 2017). Even though many of the available ground-based techniques used for the necessary investigation of plant functioning are reliable and precise, they often present downsides limiting their actual applicability. Crop water stress monitoring can be performed not only looking at the source of the problem (i.e. soil moisture conditions) but also at its effects (i.e. canopy temperature, leaf water content, etc.).

Currently, the use of remote sensing (RS) technologies for the analysis of plant water status comprises a wide range of available methods such as infrared thermometry for canopy temperature measures, microwave radiation for soil water content assessment, and spectral vegetation indices for the study of the reflectance responses of canopies to different environmental conditions. Soil optical reflection (Whiting et al., 2004 and Zhang et al., 2017), thermal emission (Verstraeten et al., 2006 and Hassan-Esfahani et al., 2015) and microwave backscatter (Das et al., 2008 and Mladenova et al., 2014) are highly correlated with soil moisture content, numerous methods for optical, thermal and microwave RS of soil moisture have been developed. Numerous studies are based on the calculation and analysis of spectral indices and have shown that there is relationship between reflectance values and canopy changes due to water stress. Measures reflectance indices within the visible (VIS) and the near-infrared (NIR) spectral range (e.g. NDVI: Normalized Difference Vegetation Index, RDVI: Renormalized Difference Vegetation Index, OSAVI: Optimized Soil Adjusted Vegetation Index) to indicate canopy changes due to water stress. Some indices (e.g. PRI: Photochemical Reflectance Index) are sensitive to the photosynthetic pigment changes due to water stress and others are used to measure the reflectance trough in the NIR and SWIR (shortwave infrared) region (e.g. SRWI: Simple Ratio Water Index and NDWI: Normalized Difference Water Index) to represent canopy moisture content. The so-called "trapezoid" or "triangle" model is one of the most widely applied approaches to RS of soil moisture utilizing both optical and thermal data. The model, hereinafter termed Thermal-Optical TRAapezoid Model (TOTRAM), is based on the interpretation of the pixel distribution within the land surface temperature-vegetation index space (Nemani et al., 1993, Carlson et al., 1994). This method and several modified versions have been used successfully for estimating surface soil moisture. With the development of sensors operating in the optical spectrum range, more and better data are being generated. Optical bands have also been incorporated into the models mentioned above. Several indices derived from the $R_{\text {red }}-R_{\text {NIR }}$ triangular space as the Perpendicular Drought Index, PDI (Ghulam et al., 2007), the Distance Drought Index, DDI (Qin et al., 2021) and the Triangle Soil Moisture Index have been proposed. Others are parameterized based on the shortwave infrared and near infrared trapezoidal space like as Perpendicular Drought Index, SPDI (Ghulam et al., 2007a) and the Modified Shortwave Infrared Perpendicular Drought Index, MSPDI (Feng et al., 2013).

Sadeghi et al., (2015) proposed a novel physically-based trapezoid model, so called OPtical TRApezoid Model (OPTRAM), which is based on a relationship between soil moisture and shortwave infrared transformed reflectance. The theoretical basis of OPTRAM, evaluate the predictive capabilities of the universally parameterized OPTRAM with Sentinel-2 and Landsat OLI observations was presented in 2017. The research presented in the article focus on the exploitation of Visible, Near Infrared and Short Wave Infrared reflectance properties for the construction of vegetation and water indices and use of the optical trapezoid model targeted to the estimation of soil water content within field. The aim of the work is to investigate the applicability of the model in the examination and mapping of the moisture content of smaller areas (agricultural field). The model ability to provide plant physiology, vegetation characteristics, and crop water status at the canopy scale can improve the site-specific decision-making process in a precision agriculture. 


\section{Materials and Methods}

The model ability to provide crop water status at the field level was tested on a relatively small agricultural area (Figure 1), that is located in Hungary. The total area of the field is 30 hectares and it is characterized by chernozem soil type. From a pedological point of view, the study area has average properties. Its organic matter content does not exceed $1-2 \%$. The annual precipitation amount is $550-650 \mathrm{~mm}$. According to the topographic map, the altitude is 302 meters above sea level at the highest point of the field and 288 meters at the lowest point that means there is a difference in height of 14 meters between the bottom and upper edge of the land. The area has varied topographic features, the west part is heavily eroded. During the study period (2017-2018) the area was covered by winter wheat.

\subsection{Satellite Images and Data Analysis}

Multispectral ESA Sentinel-2 satellite images acquired from the ESA Sentinel Scientific Data Hub were used in this study. A total of 17 cloud-free images were available, but after preliminary interpretation, 7 of them were selected for further processing. Sentinel-2 is a high spatial (10 to $60-\mathrm{m}$ ) resolution multispectral satellite having 13 spectral bands covering the visible, NIR and SWIR electromagnetic frequency domains and temporal resolution of $\sim 5$-day. The characteristics of the data used in the research include the Table 1. Workflow illustrating the main steps of data analyses for mapping surface soil moisture with OPTRAM is depicted in Figure 2. The data extraction includes the following steps: data pre-processing, multi-level image segmentation, delimitation of indices and the application of the optical trapezoid model OPTRAM. It is important to note that the Sentinel-2 Level-1C processing includes radiometric and geometric corrections including ortho-rectification and spatial registration on a global reference system with subpixel accuracy. Calculation of the TOA (the top-ofatmosphere) reflectances also occurs in this process. Hierarchical framework was used to identify the boundary of study area at super-object level and determine the unit of investigation $(20 \mathrm{~m})$ at the second level for mapping surface moisture. After pre-processing vegetation index (NDVI) and (STR) were determined. Reflectance at the red band (B4: $665 \mathrm{~nm}$ ) and the near infrared (B8: $842 \mathrm{~nm}$ ) were used to calculate the NDVI. Reflectance at the SWIR band (B12: $2190 \mathrm{~nm}$ ) was used for calculation of the STR following the Sadeghi et al., (2015).

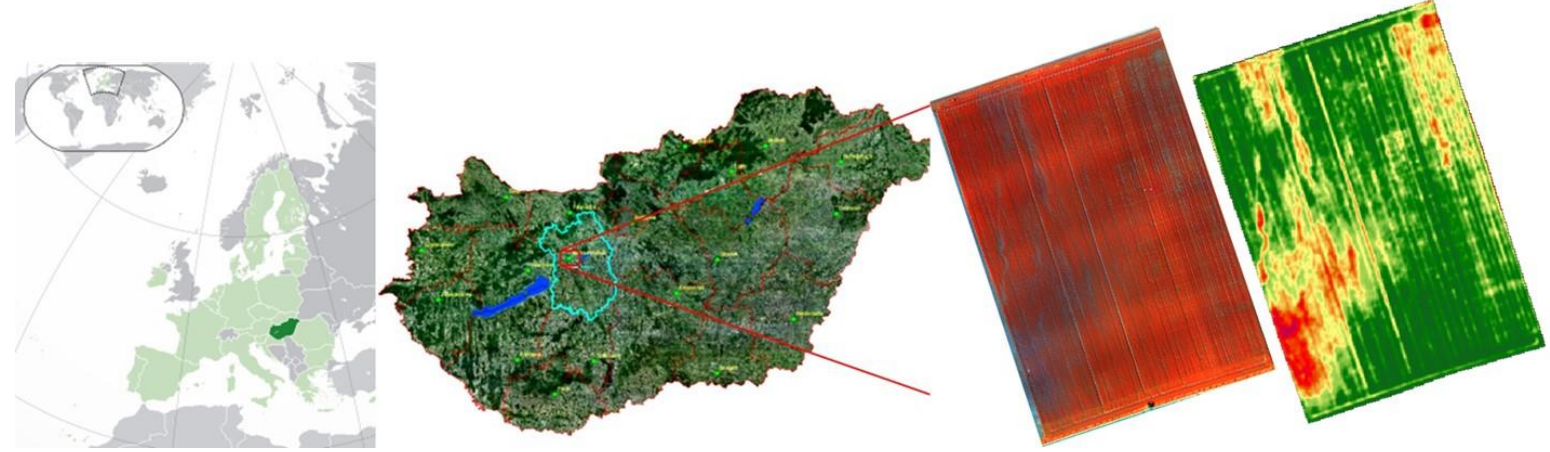

Figure 1: Location map of study area. Study area is situated in Hungry as shown to the right side. The left side is an airborne image and NDVI image. The ret colour indicates eroded area

Table 1: The specification of remote sensing data

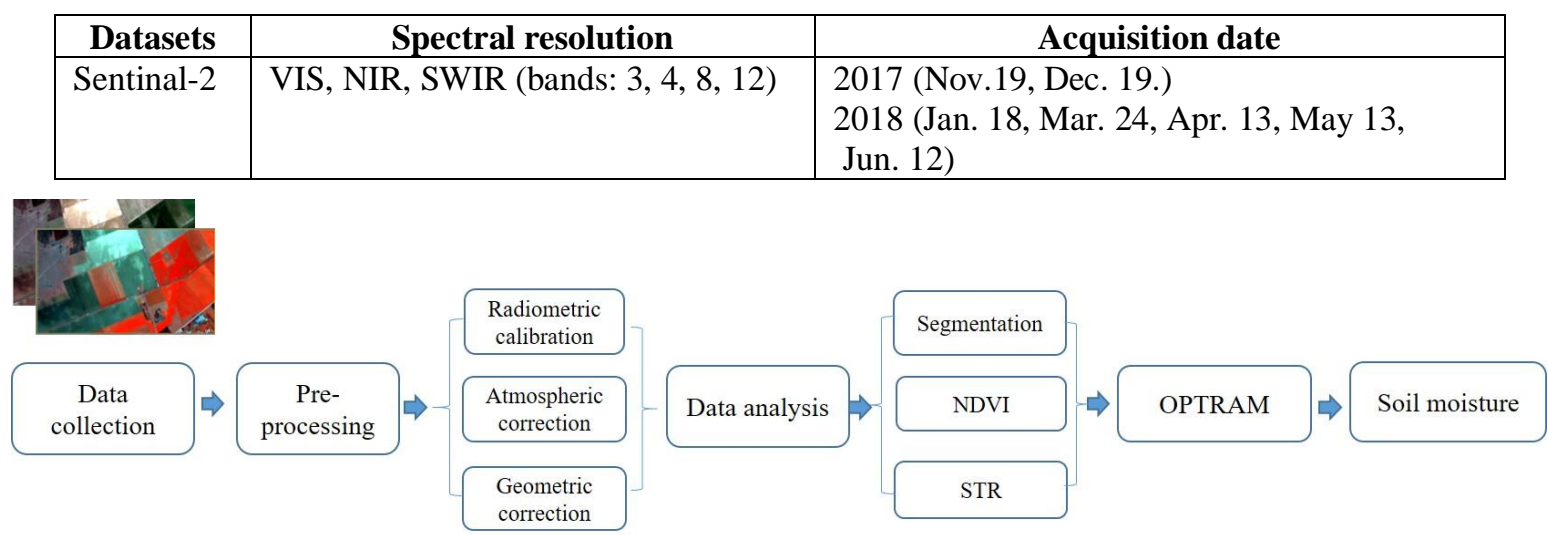

Figure 2: Satellite data analyses steps for mapping surface soil moisture with OPTRAM 
By measuring the reflectance of the plants or soil at various wavelengths, it is possible to collect a lot of information about the status of the plants and soil properties. The reflectance of light spectra depends on the land cover type (plant, soil types), water content within tissues, and other intrinsic factors. The reflectance of vegetation is low in the blue and red regions of the visible spectrum, due to absorption by chlorophyll for photosynthesis. It has a peak at the green region which gives rise to the green colour of vegetation. In the near infrared (NIR) region, the reflectance is much higher than that in the visible band due to the cellular structure in the leaves. In the mid infrared (SWIR) there are more water absorption regions. Those regions are used to examine correlation between root zone soil moisture and the vegetation status. Based on RED, NIR and SWIR bands different indices are calculated to quantify plant vigour and relate it to root zone soil moisture. The soil moisture status influences the vegetation water status and thereby changes the spectral characteristics of the vegetation (Figure 3).

The most common vegetation index used in the water stress models (e.g.TOTRAM) is the Normalized Difference Vegetation Index (NDVI). The water-absorption bands in the 1300-2500 nm region show the highest sensitivity to leaf water concentration in most crops. Short infrared is very sensitive to the water contained in the object whether it is soil or vegetation. NDWI (Normalized Difference Water Index) is one of the popular indicators of this kind of investigations. The traditional trapezoid model, TOTRAM, is based on the pixel distribution within the Land Surface Temperature-Vegetation Index space (LST-VI). An inverse linear relationship between surface soil moisture and LST is then assumed (Figure 4).

\subsection{The Optical Trapezoid Model (OPTRAM)}

The OPtical TRApezoid Model (OPTRAM) was developed for Soil Water Content (SWC) estimation making use of optical satellite data, and is based on the linear physical relationship between soil moisture and Shortwave Infrared Transformed Reflectance

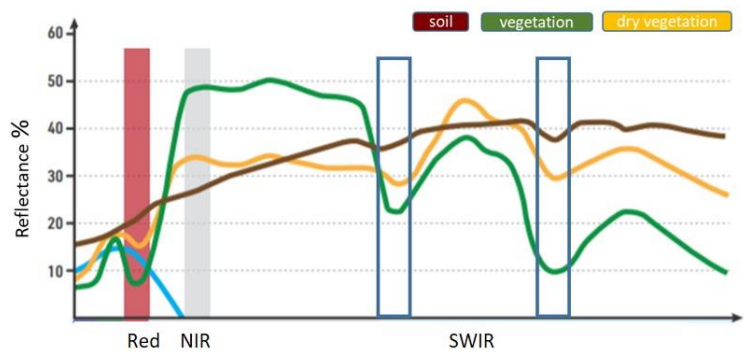

(STR) (Sadeghi, 2015). OPTRAM requires a parametrization at a given location based on the pixel distribution within STR-NDVI space (Figure 4), where STR is defined as follow:

$$
\mathrm{STR}=\left(1-\mathrm{R}_{\text {SWIR }}\right) 2 /\left(2 \mathrm{R}_{\text {SWIR }}\right)
$$

Equation 1

The model parameters can be obtained for a specific location from the dry and wet edges of the optical trapezoid depicted in (Figure 3).

$$
\begin{aligned}
& \mathrm{STR}_{\mathrm{d}}=\mathrm{i}_{\mathrm{d}}+\mathrm{S}_{\mathrm{d}} \text { NDVI } \\
& \mathrm{STR}_{\mathrm{w}}=\mathrm{i}_{\mathrm{w}}+\mathrm{s}_{\mathrm{w}} \mathrm{NDVI}
\end{aligned}
$$

Equation 2

Equation 3

where $\mathrm{STR}_{\mathrm{d}}$ and $\mathrm{STR}_{\mathrm{w}}$ are the STR at $\theta_{\mathrm{d}}$ (the local minimum dry soil moisture content) and $\theta_{\mathrm{w}}$ (the local maximum wet soil moisture content).

The soil moisture for each pixel can be estimated as a function of STR and NDVI:

$$
W=\frac{i_{d}+s_{d} N D V I-S T R}{i_{d}-i_{w}+\left(s_{d-s_{w}}\right) N D V I}
$$

Equation 4

where $i_{d}, s_{d}$, and $i_{w}, s_{w}$ are dry and wet edges parameters.

\section{Results and Discussion}

The OPTRAM model Equation (4) was parameterized based on the pixel distribution within the STR-NDVI space. The model was run separately for each available images, also one integrated trapezoid incorporating pixel distributions from all selected images was used to try universally parameterized. During the work dry (id and $s_{d}$ ) and wet $\left(i_{w}\right.$ and $\left.s_{w}\right)$ edges were determined by visual inspection of the STR-NDVI spaces so that the trapezoids surrounded the majority of the pixels (Table 2).

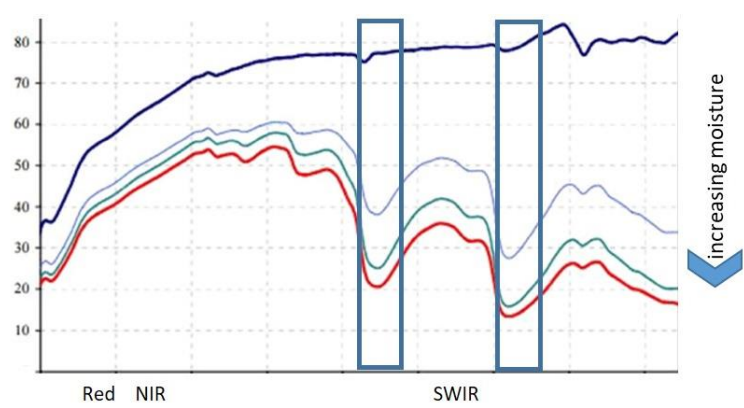

Figure 3: Spectral reflectance of vegetation and soil with different levels of water content (yellow line: dry vegetation). The spectral bands used to calculate the indices are highlighted 

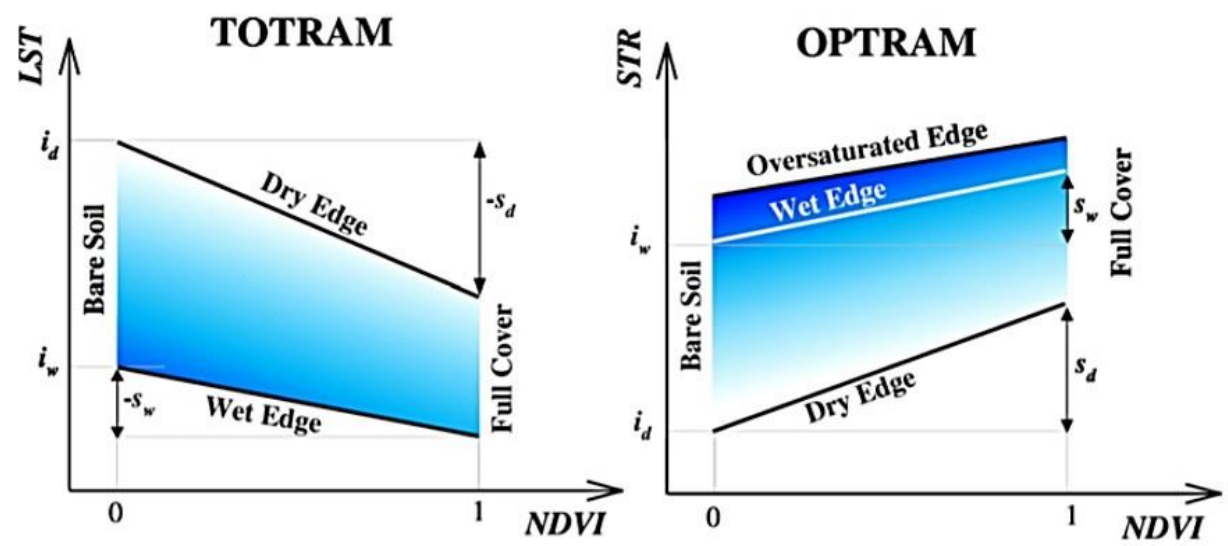

Figure 4: Sketch illustrating parameters of the traditional thermal-optical trapezoid model (TOTRAM) and the new optical trapezoid model (OPTRAM) (Sadeghi, 2015)

Table 2: OPTRAM parameters obtained for the study area (agricultural field) based on Sentinel-2 (2018)

\begin{tabular}{|c|c|c|c|c|}
\hline Data & Dry edge $\mathbf{i}_{\mathbf{d}}$ & Dry edge $\mathbf{S}_{\mathbf{d}}$ & ${\text { Wet edge } \mathbf{i}_{\mathbf{w}}}$ & Wet edge $\mathbf{S}_{\mathbf{w}}$ \\
\hline 2018.01 .18 & 3.8 & 1.2 & 4.6 & 0.9 \\
\hline 2018.04 .08 & 0 & 0.5 & 1.8 & 2.9 \\
\hline 2018.06 .12 & 3.5 & 1 & 4.6 & 2.4 \\
\hline
\end{tabular}
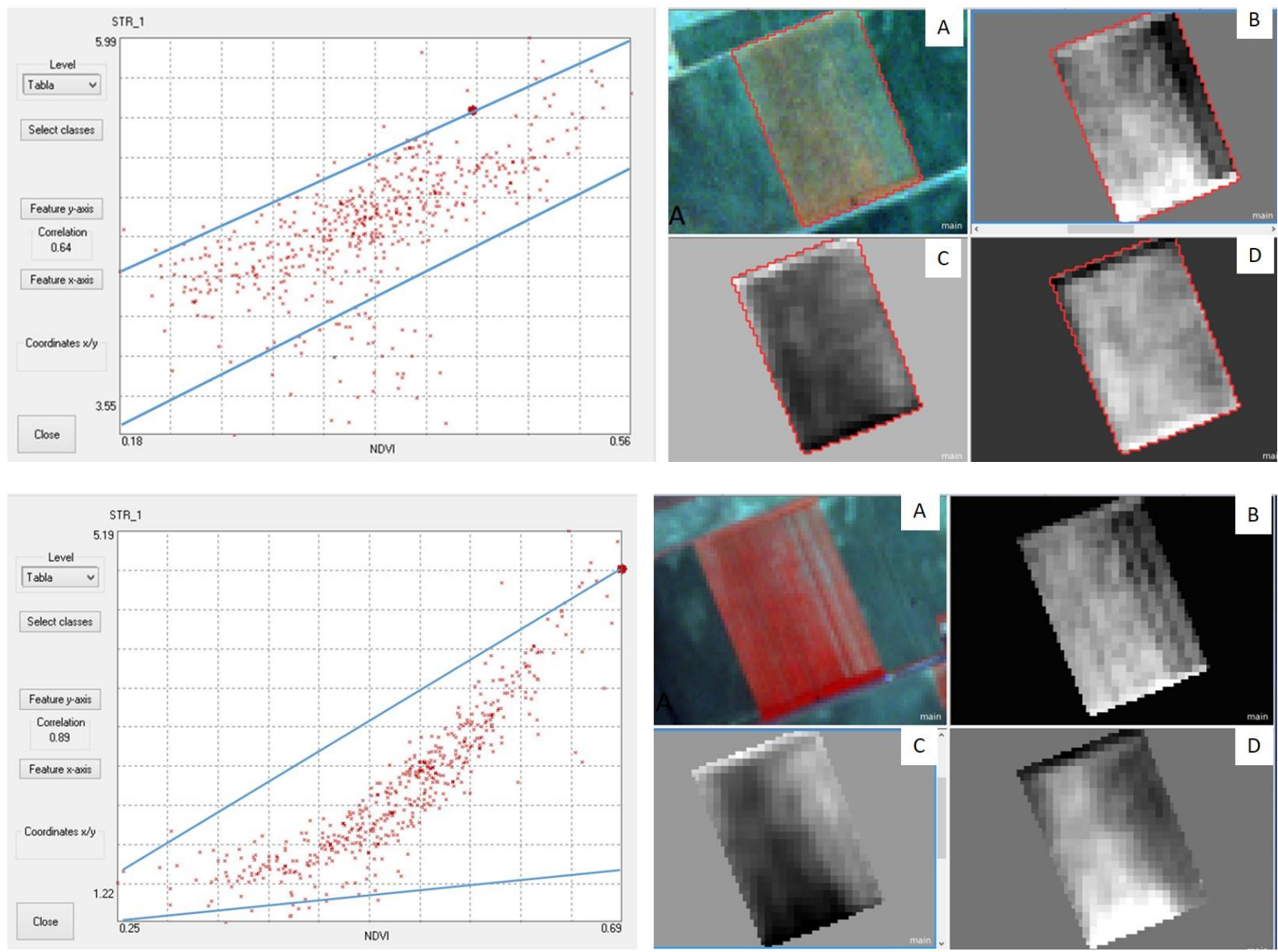

Figure 5: Pixel distributions within the STR-NDVI space for images (2018 Jan., Apr. and Jul) A: false colour composition, B: NDVI, C: B12 values, D: W values (OPTRAM) (Continune Next Page) 

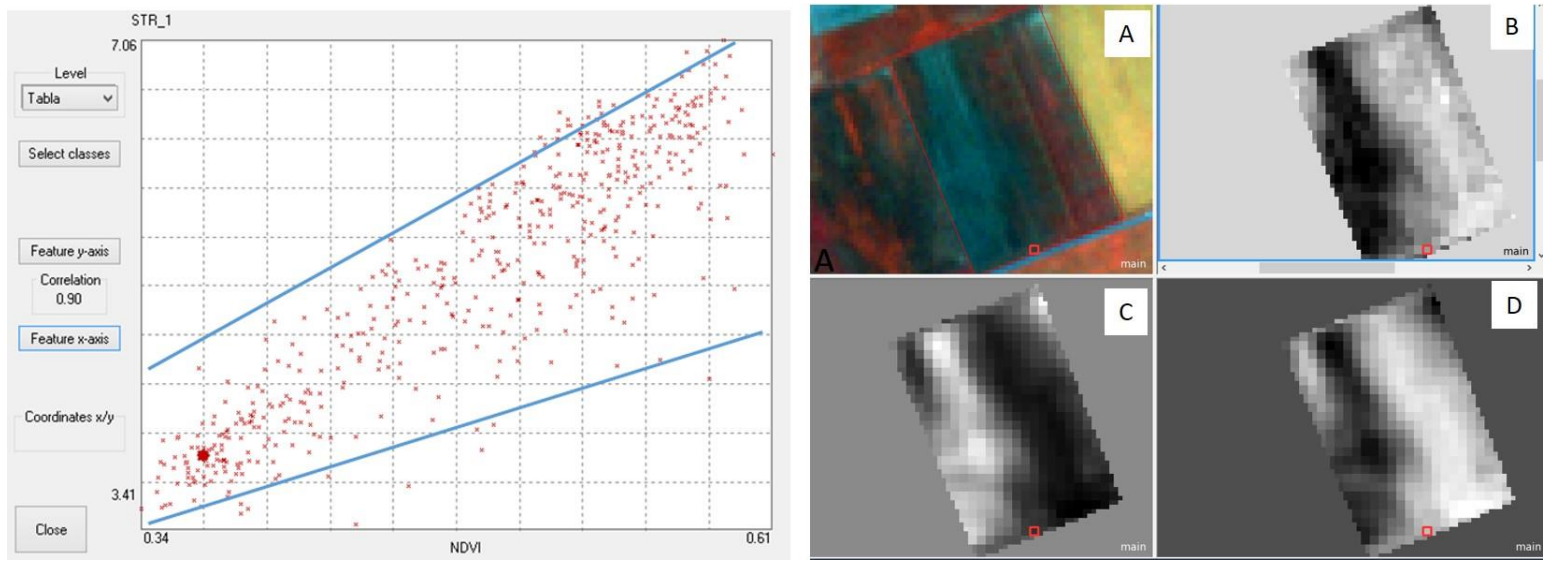

Figure 5: Pixel distributions within the STR-NDVI space for images (2018 Jan., Apr. and Jul) A: false colour composition, B: NDVI, C: B12 values, D: W values (OPTRAM)

From id and sd (dry edge parameters) and iw and siw (wet edge parameters), the normalized moisture content, W, was estimated for each pixel with Equations $(1,2,3,4)$. The results are illustrated in the Figure 5. Pixel distributions within the STR-NDVI space for 3 images (as example) are depicted in the Figure 5. Corresponding model parameters are listed in Table 2. According to the study results a trapezoidal shapes were formed by the pixels in the STR-NDVI space in all cases. The normalized moisture content (W), was estimated for each images. Soil moisture variability within the field are clearly detected by using this model. However, farreaching conclusions cannot be drawn. Further research is needed. Currently, additional agricultural areas have been included in the research, and devices suitable for meteorological measurements have been placed in the area. Field measurements are absolutely necessary to continue the study and validate the results.

\section{References}

Carlson, T. N., Gillies, R. R. and Perry, E. M., 1994, A Method To Make Use Of Thermal Infrared Temperature and NDVI Measurements to Infer Surface Soil Water Content and Fractional Vegetation Cover. Remote Sensing Reviews. Vol. 9(1-2), 161-173.

Das, N. N., Mohanty, B. P. and Njoku, E. G., 2008, Characterization of Backscatter by Surface Features in L-band Active Microwave Remote Sensing of Soil Moisture. IGARSS 2008IEEE International Geoscience and Remote Sensing Symposium. 2 IEE E, II-817.

Feng, H., Chen, C., Dong, H., Wang, J. and Meng, Q., 2013, Modified Shortwave Infrared Perpendicular Water Stress Index: A Farmland Water Stress Monitoring Method. Journal of
Applied Meteorology and Climatology. Vol. 52(9), 2024-2032.

Ghulam, A., Qin, Q. and Zhan, Z., 2007, Designing of the Perpendicular Drought Index. Environmental Geology. Vol. 52(6), 1045-1052.

Ghulam, A., Li, Z. L., Qin, Q., Tong, Q., Wang, J., Kasimu, A. and Zhu, L., 2007a, A Method for Canopy Water Content Estimation for Highly Vegetated Surfaces-Shortwave Infrared Perpendicular Water Stress Index. Science China Earth Sciences. Vol. 50(9), 1359-1368.

González-Dugo, M., Moran, M., Mateos, L. and Bryant, R., 2006, Canopy Temperature Variability as an Indicator Of Crop Water Stress Severity, Irrigation Science, Vol. 24, 233-240.

Hassan-Esfahani, L., Torres-Rua, A., Jensen, A. and McKee, M., 2015, Assessment of Surface Soil Moisture Using High-Resolution Multi-Spectral Imagery and Artificial Neural Networks. Remote Sensing. Vol. 7(3), 2627-2646.

Ihuoma, O. S. and Madramootoo, Ch. A., 2017, Recent Advances in Crop Water Stress Detection, Computers and Electronics in Agriculture, Vol. 141, 267-275.

Jones, H., 2012, Remote Sensing of Plant Stresses and its Use in Irrigation Management, VII International Symposium on Irrigation of Horticultural Crops, Vol. 1038 , 239-247.

Mladenova, I. E., Jackson, T. J., Njoku, E., Bindlish, R. Chan, S., Cosh, M. H., Holmes, T. R. H., De Jeu, R. A. M., Jones, L., Kimball, J. and Paloscia, S., 2014, Remote Monitoring of Soil Moisture Using Passive Microwave-Based TechniquesTheoretical Basis and Overview of Selected Algorithms for AMSR-E. Remote Sensing of Environment. Vol. 144, 197-213.

Nemani, R., Pierce, L., Running and Goward, S., S., 1993, Developing Satellite-Derived Estimates of Surface Moisture Status. Journal of Applied 
Meteorology and Climatology, Vol. 32 (3), 548557.

Osakabe, Y., Osakabe, K., Shinozaki, K. and LamSon, P., 2014, Response of Plants to Water Stress, Frontiers in Plant Science, Vol. 5(86), 18, https://doi.org/10.3389/fpls.2014.00086.

Osroosh, Y., Peters, R. T., Campbell, C. S. and Zhang, Q., 2015, Automatic Irrigation Scheduling of Apple Trees Using Theoretical Crop Water Stress Index with an Innovative Dynamic Threshold. Computers and Electronics in Agriculture, Vol. 118, 193-203.

Sadeghi, M., Jones, S. B. and Philpot, W. D., 2015, A Linear Physically-Based Model for Remote Sensing of Soil Moisture Using Short Wave Infrared Bands. Remote Sensing of Environment. Vol. 164, 66-76.

Qin, Q., Jin, C., Zhang, N. and Yang, X., 2010, An Two-Dimensional Spectral Space Based Model for Drought Monitoring and its Re-Examination. Geoscience and Remote Sensing Symposium (IGARSS), 2010 IEEE International. IEEE, 38693872 .
Verstraeten, W., Veroustraete, W. F., van der Sande, C. J., Grootaers, I. and Feyen, J., 2006, Soil Moisture Retrieval Using Thermal Inertia, Determined with Visible and Thermal Spaceborne Data, Validated for European Forests. Remote Sensing of Environment. Vol. 101(3), 299-314.

Wang, X., Zhao, C., Guo, N., Li, Y., Jian, S. and Yu, K., 2015, Determining the Canopy Water Stress for Spring Wheat using Canopy Hyperspectral Reflectance Data in Loess Plateau Semiarid Regions. Spectroscopy Letters, Vol. 48, 492-498.

Whiting, M. L., Li, L. and Ustin, S. L., 2004, Predicting Water Content Using Gaussian Model on Soil Spectra. Remote Sensing of Environment. Vol. 89(4), 535-552.

Zhang, H., Han, M., Chavez J.L. and Lan, Y., 2017, Improvement in Estimation of Soil Water Deficit by Integrating Airborne Imagery Data into a Soil Water Balance Model. International Journal of Agricultural and Biological Engineering, Vol. 10(3), 37-46. 